\title{
Acid sphingomyelinase-mediated blood-brain barrier disruption in aging
}

\author{
Min Hee Park ${ }^{1,2,3}$, Hee Kyung Jin ${ }^{1,4, *} \mathcal{E}$ Jae-sung Bae $e^{1,2,3, *}$ \\ ${ }^{1}$ KNU Alzheimer's disease Research Institute, Kyungpook National University, Daegu 41566, ${ }^{2}$ Department of Physiology, Cell and Matrix \\ Research Institute, School of Medicine, Kyungpook National University, Daegu 41944, ${ }^{3}$ Department of Biomedical Science, BK21 Plus \\ KNU Biomedical Convergence Program, Kyungpook National University, Daegu 41944, ${ }^{4}$ Department of Laboratory Animal Medicine, \\ College of Veterinary Medicine, Kyungpook National University, Daegu 41566, Korea
}

\begin{abstract}
Although many studies have reported that the breakdown of the blood-brain barrier (BBB) represents one of the major pathological changes in aging, the mechanism underlying this process remains relatively unexplored. In this study, we described that acid sphingomyelinase (ASM) derived from endothelial cells plays a critical role in BBB disruption in aging. ASM levels were elevated in the brain endothelium and plasma of aged humans and mice, resulting in BBB leakage through an increase in caveolae-mediated transcytosis. Moreover, ASM caused damage to the caveolae-cytoskeleton via protein phosphatase 1-mediated ezrin/radixin/moesin dephosphorylation in primary mouse brain endothelial cells. Mice overexpressing brain endothelial cell-specific ASM exhibited acceleration of BBB impairment and neuronal dysfunction. However, genetic inhibition and endothelial specific knock-down of ASM in mice improved BBB disruption and neurocognitive impairment during aging. Results of this study revealed a novel role of ASM in the regulation of BBB integrity and neuronal function in aging, thus highlighting the potential of ASM as a new therapeutic target for anti-aging. [BMB Reports: Perspective 2019; 52(2): 111-112]
\end{abstract}

*Corresponding authors. Jae-sung Bae, E-mail: jsbae@knu.ac.kr; Hee Kyung Jin, E-mail: hkjin@knu.ac.kr

https://doi.org/10.5483/BMBRep.2019.52.2.033

\section{Received 11 January 2019}

Keywords: Acid sphingomyelinase, Aging, Blood-brain barrier, Caveolae, Endothelial cell

Abbreviations: ASM, Acid sphingomyelinase; BBB, Blood-brain barrier; Cav-1, Caveolin-1; ERM, Ezrin/radixin/moesin; PP1, Protein phosphatase 1

Perspective to: Min Hee Park et al. (2018), Vascular and neurogenic rejuvenation in aging mice by modulation of ASM, Neuron, 100(1):167-182.e9. doi: 10.1016/j.neuron.2018.09.010.
Aging is related to progressive deterioration of central nervous system function and contributes to the pathogenesis of neurodegenerative disease. Previous studies have implicated alterations in the molecular mechanisms of aging with changes in the brain environment such as abnormal aggregation of proteins, neuronal loss, neuroinflammation, and cognitive deficits. The dysfunction(s) in aging could lead to neurodegenerative disease including mild cognitive impairment, cerebrovascular disease, Parkinson's disease and Alzheimer's disease. In particular, disruption of the blood-brain barrier (BBB) is one of several major pathological features of these age-related neurodegenerative diseases. Moreover, many studies have demonstrated that BBB dysfunction may be a cause or consequence of neurodegeneration.

Acid sphingomyelinase (ASM), encoded by the Smpd1 gene, plays an important housekeeping role in sphingolipid metabolism and is known to regulate cell apoptosis, proliferation, and differentiation. Although ASM is expressed in virtually all cell types under normal conditions, ASM secreted from endothelial cells (ECs) is significantly associated with numerous diseases. ECs are the major cell type forming the $\mathrm{BBB}$, along with pericytes and astrocytes, and the interaction between ECs and other neuronal cells is critical for the maintenance of neurological health in the brain. Previous studies have suggested that an increase in ASM activity may contribute to age-related brain damage. Nevertheless, the specific role of ASM in maintaining the integrity of the BBB and/or age-related neurodegeneration remains unclear. In a recent study published in Neuron, we for the first time demonstrated that ASM derived from ECs plays a central role in aging-induced BBB disruption and neurodegeneration.

Higher ASM activity was detected in plasma from older individuals (65-90 years) compared with plasma from their younger counterparts (24-45 years). We also confirmed similar results in plasma derived from old mice (20-months). Interestingly, ASM activity was higher in the brain tissues of old mice than in other organs such as the liver, kidney, spleen, heart, lung, stomach, genitals, muscle and fat, compared with younger mice. The robust elevation in ASM levels in the brains of old mice was associated with microvessels, and ECs derived 
from microvessels were the main contributors for elevated ASM activity. These results indicated that ASM activity increased in aged plasma and brain ECs, and could affect brain dysfunction in the process of aging. Old Smpd $1^{+/-}$mice, in which ASM is genetically inhibited, exhibited a significant reduction in ASM activity in the plasma and brain ECs. In addition, capillary density in the brains of older mice was decreased by EC death, while old Smpd $1^{+/-}$mice exhibited higher capillary density. BBB permeability was also increased in the brains of old mice. In contrast, a substantial decrease in permeability in old $S m p d 1^{+/-}$mice was observed. However, pericyte coverage, astrocytic end-foot processes, and junctional properties, which are responsible for the restoration of BBB integrity, exhibited no differences in the brains of old mice and old Smpd $1^{+/-}$mice. Moreover, the leakiest vessels in the brain did not exhibit an apoptotic signal in both old mice and old Smpd $1^{+/-}$mice, indicating that the death of ECs caused by ASM was not the main cause of BBB disruption in aging.

The transcellular transport pathway (transcytosis) is known to regulate BBB permeability. Recently, an increase in the number of caveolae, a vesicle carrier involved in transcytosis, has been reported to increase in BBB permeability. Caveolin-1 (Cav-1) is the main structural protein in caveolae and phosphorylation of Cav-1 is an indicator of endothelial transcytosis levels. We confirmed that Cav-1 phosphorylation was elevated in vessels isolated from the brains of older mice; however, reduction in Cav-1 level was observed in samples from old $S m p d 1^{+/-}$mice. Furthermore, a significant increase in the number of Cav-1-positive vesicles was observed in ECs from the brains of old mice and a decrease in the number was found in old Smpd1 $1^{+/-}$mice. These results suggested that ASM regulated caveolae-mediated transcytosis in the aging brain. Interactions between caveolae and the actin cytoskeleton contribute to maintaining cell membrane integrity. Moreover, ezrin/radixin/moesin (ERM) proteins, which interact with the cytoskeleton, are involved in linking the cell membrane. ASM-treated primary mouse brain ECs exhibited a loss of actin filaments and dephosphorylation of ERM. This ASM-induced ERM dephosphorylation was triggered by the activation of protein phosphatase 1 (PP1). Collectively, these findings suggest that ASM causes disruption of the cytoskeleton through PP1-mediated ERM dephosphorylation and leads to internalization of caveolae, resulting in an increase in BBB permeability.

Slco1c1-CreERT2;Smpd1 ${ }^{\text {ox/ox }}$ mice, which overexpress brain EC-specific ASM, exhibited BBB leakage through caveolaemediated transcytosis and ERM dephosphorylation only after 13 months of age. Interestingly, these mice exhibited a significant reduction in the number of neuronal cells in the brain and severe memory impairments compared with age-matched control mice. These results suggested that ASM derived from the brain endothelium accelerated BBB disruption and evoked aging-like brain pathology. Administration of EC-specific green fluorescent protein-labeled Smpd1 miR RNAi led to selective suppression of ASM activity in the plasma and brain microvessels of both aged S/Co1c1-CreERT2;Smpd1 $1^{\text {ox/ox }}$ mice and old mice. Moreover, Smpd1 miR RNAi treatment resulted in the reduction in caveolae-mediated transcytosis and ERM dephosphorylation in the microvessels of the brains of these mice. Furthermore, neuronal density and behavioral function were recovered after Smpd1 miR RNAi treatment. Collectively, these results highlighted that brain EC-derived ASM played a critical role in the regulation of aging brain pathology; thus, inhibition of endothelial ASM mitigated the negative effects of aging by controlling BBB integrity and neuronal function.

In conclusion, our findings suggest a central role for ASM as a regulator of BBB integrity and neuronal function in aging, as well as highlight the potential of ASM as a drug target for anti-aging. To date, few inhibitors that can directly inhibit ASM have been found; nevertheless, highly potent and selective ASM inhibitors are anticipated to be developed in the future as the crystal structure of this enzyme is revealed. Therefore, further studies investigating the development of functional ASM inhibitors may be highly valuable for understanding the anti-aging process and for the treatment of various age-related neurodegenerative diseases.

\section{ACKNOWLEDGEMENTS}

This work was supported by the National Research Foundation (NRF) grant funded by the Korea government (MSIT) (2017R 1A2A1A17069686, 2017R1A4A1015652 and 2018M3C7A10 56513). 